Meta

Journal des traducteurs

Translators' Journal

\title{
La nomenclature des composés organiques
}

\section{Gilles Bélanger}

Volume 28, numéro 2, juin 1983

URI : https://id.erudit.org/iderudit/002042ar

DOI : https://doi.org/10.7202/002042ar

Aller au sommaire du numéro

Éditeur(s)

Les Presses de l'Université de Montréal

ISSN

0026-0452 (imprimé)

1492-1421 (numérique)

Découvrir la revue

Citer cet article

Bélanger, G. (1983). La nomenclature des composés organiques. Meta, 28(2),

166-172. https://doi.org/10.7202/002042ar d'utilisation que vous pouvez consulter en ligne.

https://apropos.erudit.org/fr/usagers/politique-dutilisation/ 


\section{LA NOMENCLATURE DES COMPOSÉS ORGANIQUES}

La chimie organique étudie les composés du carbone et de l'hydrogène, y compris ceux qui renferment des hétéro-atomes, tels l'oxygène, l'azote, le soufre, le phosphore, les halogènes et certains métaux. Aucun autre élément ne donne autant de composés que le carbone, et les possibilités de synthèse de nouveaux corps sont loin d'être épuisées. À la difficulté de nommer ces composés, en raison de leur multiplicité et de leur complexité, s'ajoute celle des nombreux synonymes que l'on peut proposer pour un même corps. En effet, nommer un corps chimique consiste à le décrire complètement, et les résultats peuvent être forts différents suivant que l'on adopte l'une ou l'autre des méthodes de nomenclature acceptées. Heureusement, il est généralement possible de dégager des structures fondamentales, relativement peu nombreuses, qui conduisent à établir des séries de composés ne différant que par quelques caractéristiques secondaires, ce qui simplifie d'autant leur nomenclature. Ces structures fondamentales sont essentiellement des chaînes carbonées ouvertes (acycliques) ou fermées (cycliques), non ramifiées, qui incluent parfois des hétéro-atomes et sur lesquelles se greffent des chaînes latérales et divers substituants.

Un corps dans lequel un hétéro-atome se substitue à un atome d'hydrogène (par une réaction de substitution réelle ou hypothétique) conserve son nom fondamental, modifié toutefois pour tenir compte de la substitution. Dans d'autres cas, on considère qu'un corps résulte de l'addition d'un atome ou d'une molécule à une autre molécule, de la soustraction d'atomes ou de groupes d'atomes, de la fusion de deux ou plusieurs molécules, etc. Suivant le type de réaction, différentes méthodes de nomenclature peuvent donc être adoptées. On trouvera ci-dessous quelques exemples illustrant la façon dont sont construits les noms systématiques en chimie organique. Quant aux règles particulières de nomenclature, établies par la Commission de nomenclature pour la chimie organique de l'Union internationale de chimie pure et appliquée, on en trouvera un exposé détaillé dans les ouvrages mentionnés dans la bibliographie.

Dans un précédent article ${ }^{1}$, nous avons déjà dit que la nomenclature chimique se compose de noms triviaux (trivial name) tels chlore ou quinine, de noms semi-triviaux (semi-trivial name, semi-systematic name), tels méthane ou benzène, et de noms systématiques (systematic name), tels pentane ou hexane. Par ailleurs, ces noms sont dits fondamentaux s'ils désignent des corps considérés comme structures à partir desquelles sont formés d'autres corps, ou dérivés s'ils sont obtenus en adjoignant à des noms fondamentaux un ou plusieurs affixes. Chlorobenzène et pentadiène sont des noms dérivés de noms fondamentaux (semi-trivial dans le premier cas, systématique dans le second). C'est évidemment dans les noms dérivés que se trouvent les plus grandes difficultés de traduction.

1. Meta, vol. 27, $\mathrm{n}^{\circ}$ 3, septembre 1982, p. 295-305. 


\section{A) MÉTHODES DE NOMENCLATURE}

1. La nomenclature substitutive (substitutive nomenclature), de loin la plus courante, sert à nommer les corps résultant de réactions de substitution, réelles ou hypothétiques. Des atomes d'hydrogène d'un composé fondamental (parent compound) peuvent être remplacés par divers groupes caractéristiques (characteristic group) donnant à la nouvelle molécule ses propriétés physico-chimiques. Ces groupes sont formés de substituants (substituent), que l'on peut classer en substituants inorganiques (tels les halogènes) et en substituants fonctionnels ${ }^{2}$. Que la réaction de substitution soit possible ou non, on considère que ces groupes caractéristiques se substituent à des atomes d'hydrogène sur le squelette de la molécule (la chaîne carbonée).

Dans un nom substitutif, un seul groupe caractéristique peut être choisi comme suffixe modifiant le nom fondamental. Les groupes fonctionnels sont donc classés en groupes principaux et en groupes secondaires, suivant un ordre de priorité établi par les règles de nomenclature et qui est le même en anglais et en français. Une fois choisi le groupe principal déterminant le suffixe, on indique tous les autres groupes à l'aide de préfixes. Les substituants inorganiques sont par ailleurs toujours désignés par des préfixes dans les noms substitutifs. Tous les éléments du nom dérivé sont en général soudés si l'on n'indique pas les indices de position (locant) des substituants.

hydroxypentanone or<smiles>CC(=O)CCCO</smiles>
5-hydroxy-2-pentanone

hydroxypentanone ou hydroxy-5 pentanone-2

Le suffixe -one dénote la présence du groupe $>(\mathrm{C})=0$, qui a priorité sur le groupe - $\mathrm{OH}$, indiqué par le préfixe hydroxy-. Notons que les indices de position se placent après l'affixe en français, et avant l'affixe en anglais (avec des traits d'union entre tous les éléments du nom). Dans l'exemple qui suit, le groupe -OH est prioritaire :

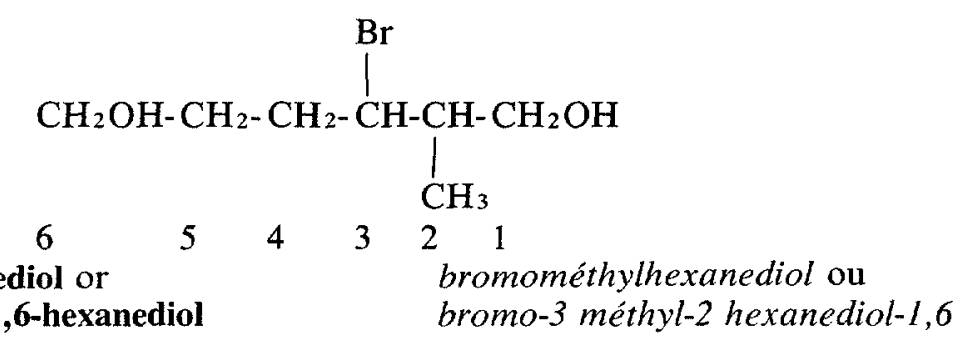

bromomethylhexanediol or bromo-3 méthyl-2 hexanediol-1,6

2. On appelle fonction (function) l'ensemble des propriétés physico-chimiques que confère à une molécule un assemblage d'atomes renfermant au moins un hétéro-atome (hetero-atom); cet assemblage porte le nom de groupement ou groupe fonctionnel et, par extension, de fonction (functional group, function). En anglais, le sens de function est plus large et s'étend par exemple aux doubles et triples liaisons à l'intérieur de la chaîne carbonée (carbon chain). Le groupe fonctionnel comprend le ou les substituants fonctionnels et l'atome de carbone qui le porte. Par exemple, la fonction acide carboxylique provient du groupement fonctionnel $-\mathrm{COOH}$, dont les substituants fonctionnels sont $-\mathrm{OH}$ et $=\mathrm{O}$. 
2. La nomenclature radico-fonctionnelle (radicofunctional nomenclature) ne convient qu'aux corps renfermant une seule fonction. Le groupe caractéristique est exprimé par un nom de fonction (functional class name) du genre alcool, cétone, éther-oxyde, sulfure, chlorure, etc. En français, le nom de la fonction est suivi du nom du radical, à l'inverse de l'anglais.

methyl alcohol

$\mathrm{CH}_{3} \mathrm{OH}$

alcool méthylique

Dans la nomenclature substitutive, le nom de ce composé est méthanol.

ethyl oxide or

$\mathrm{C}_{2} \mathrm{H}_{5}-\mathrm{O}-\mathrm{C}_{2} \mathrm{H}_{5}$

oxyde d'éthyle ou

diethyl ether

éther-oxyde d'éthyle ou

éther diéthylique

Les noms des radicaux peuvent aussi être préfixés au nom de la fonction, comme en anglais :

ethyl methyl ketone<smiles>CCC(C)=O</smiles>

méthyl-éthyl-cétone

3. La nomenclature additive (additive nomenclature) correspond à des réactions d'addition, réelles ou hypothétiques, sur des corps insaturés. Dans ce cas, on nomme les atomes ou groupes d'atomes ajoutés à la structure fondamentale. Les noms additifs fonctionnels sont du même type que les noms radicofonctionnels.

ethylene oxide

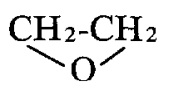

oxyde d'éthylène

La double liaison du composé de départ $\left(\mathrm{CH}_{2}=\mathrm{CH}_{2}\right)$ a été brisée par l'addition d'un atome d'oxygène.

ethylene dichloride<smiles>ClCCCl</smiles>

dichlorure d'éthylène

Suivant la méthode substitutive, ce corps s'appelle dichloréthane. L'addition d'hydrogène s'indique par le préfixe hydro:

\section{1,2,3,4-tetrahydronaphthalene}<smiles>c1ccc2c(c1)CCCC2</smiles>

tétrahydro-1,2,3,4 naphtalène

Les combinaisons organo-métalliques ont aussi des noms additifs :

trimethylaluminum phenylmercury chloride
$\mathrm{Al}\left(\mathrm{CH}_{3}\right)_{3}$

$\mathrm{C}_{6} \mathrm{H}_{5} \mathrm{HgCl}$ triméthyl-aluminium chlorure de phényl-mercure

4. La nomenclature soustractive (substractive nomenclature) sert à indiquer l'insaturation ou l'élimination d'atomes ou de groupes d'atomes de la structure fondamentale. Les noms soustractifs comportent :

a) des préfixes indiquant l'élimination
i) d'eau
ii) d'hydrogène
anhydro-
anhydro-
iii) d'oxygène
dehydro-
déhydro-
deoxy-
désoxy- 
iv) de groupes méthyl ou méthylène

b) des suffixes indiquant

i) I'insaturation

ii) l'élimination d'eau nor-

$\begin{array}{ll}\text { ene } & \text { ène } \\ \text { yne } & \text { yne } \\ \text { olactone } & \text { olactone } \\ \text { olide } & \text { olide }\end{array}$

c) des noms de fonction (anhydride, lactone, etc.).

acetic anhydride

$\mathrm{CH}_{3}-\mathrm{CO}-\mathrm{O}-\mathrm{CO}-\mathrm{CH}_{3}$

butyrolactone

deoxyribose

$\mathrm{HOCH}_{2}$<smiles>OC1CC2(O)CC1O2</smiles>

norpinane<smiles>O=C1CCCO1</smiles>

anhydride acétique

butyrolactone

désoxyribose

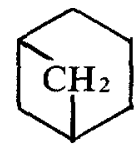

norpinane

5. La nomenclature conjonctive (conjunctive nomenclature) juxtapose les noms fondamentaux de deux composés, l'un cyclique, l'autre aliphatique, réunis par élimination d'un hydrogène sur chaque composé. Le nom du composé cyclique précède celui du composé aliphatique.

naphthaleneacetic acid

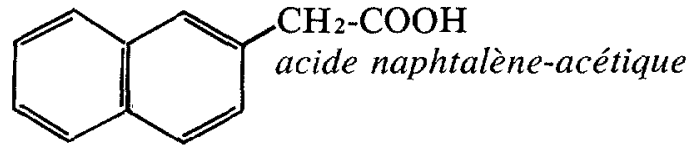

cyclohexanecarbodithionic acid

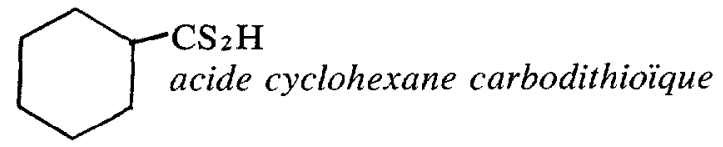

6. La nomenclature par remplacement, ou nomenclature en « $a$ " (replacement nomenclature, «a» nomenclature) sert à nommer des hydrocarbures cycliques et acycliques comportant dans leur chaine carbonée des hétéro-atomes. On l'utilise lorsque les autres méthodes de nomenclature ne permettent pas de constituer des noms systématiques simples. Pour former des noms en « $a$, on considère que la chaîne carbonée comprend aussi les hétéro-atomes placés entre deux atomes de carbone (mais non ceux qui remplacent des atomes d'hydrogène, ceux-ci étant toujours traités comme des groupes fonctionnels). Les hétéro-atomes de la chaîne carbonée sont nommés à l'aide de préfixes en «a»: oxa-, aza-, thia-, etc.

$$
\begin{array}{ccccccccccc}
\mathrm{NO}_{2}-\mathrm{CH}_{3}-\mathrm{O}-\mathrm{CH}_{2}-\mathrm{O}-\mathrm{CH}_{2}-\mathrm{CH}_{2}-\mathrm{O}-\mathrm{CH}_{2}-\mathrm{CH}_{2}-\mathrm{O}-\mathrm{CH}_{3} \\
11 & 10 & 9 & 8 & 7 & 6 & 5 & 4 & 3 & 2 & 1
\end{array}
$$

11-nitro-2,5,8,10-tetraoxaundecane nitro-11 tétraoxa-2,5,8,10 undécane 


$$
\begin{array}{llllll}
\mathrm{CH}_{3}-\mathrm{CHCl}-\mathrm{O}-\left[\mathrm{CH}_{2}\right]_{2}-\mathrm{NH}-\left[\mathrm{CH}_{2}\right]_{2}-\mathrm{O}-\left[\mathrm{CH}_{2}\right]_{2}-\mathrm{S}-\mathrm{CH}_{2}-\mathrm{COOH} \\
14 & 13 & 12 & 9 & 6 & 32
\end{array}
$$

13-chloro-6,12-dioxa-3-thia-9-

acide chloro-13 dioxa-6,12 thia-3 azatetradecanoic acid

aza-9 tétradecanoïque

7. Les hétérocycles condensés (fused heterocyclic system), c'est-à-dire les molécules formées d'au moins deux cycles, partageant au moins deux atomes de carbone et dont un cycle au moins comporte un ou des hétéro-atomes, reçoivent des noms de fusion (fusion name), dans lesquels le nom du premier cycle nommé est modifié en «O».

benzofuran

benzothiophene<smiles></smiles>

benzofuranne

benzothiophène

Bon nombre de ces hétérocycles ont toutefois reçu des noms triviaux, tels xanthène, carbazole, purine, etc. Par ailleurs, les préfixes en «O» s'appliquent aussi à d'autres systèmes polycycliques.

Pour établir le nom d'un composé acyclique, on choisit d'abord la chaîne carbonée la plus longue et qui donne le moins possible de groupes fonctionnels dans les chaînes latérales (side chain). Les substituants, chaînes latérales, doubles et triples liaisons, etc., sont ensuite indiqués au moyen d'affixes. Dans le cas des composés cycliques, on précise d'abord la structure d'ensemble, puis on indique la nature et la position des groupes fonctionnels.

\section{B) LES NOMBRES DANS LES NOMS CHIMIQUES}

Les nombres figurant dans les noms chimiques sont de deux types. Des affixes numériques littéraux (multiplying affix) indiquent le nombre d'atomes, de groupes fonctionnels ou d'éléments de structure que renferme la molécule; les positions de ces atomes, groupes fonctionnels ou éléments de structure sont représentées par des chiffres arabes.

\section{Affixes numériques littéraux}

Il existe quatre séries d'affixes littéraux.

a) La série énumérative, formée de préfixes grecs $(d i$, tri, tétra,...) est utilisée pour indiquer le nombre d'atomes de carbone dans un alcane ou un cycle (pentalène, hexane), les substitutions (pentachloro-) et les insaturations (butadiène). Bi peut être substitué à $d i$ dans certains noms (biphényle, bicyclo). Les préfixes composés se construisent dans l'ordre inverse du français et de l'anglais : 132 = dotriacontahecta. Do remplace di dans les préfixes composés et undéca (onze) est préféré à hendéca. Dans les préfixes supérieurs, on conserve hen (un).

b) La série multiplicative (bis, tris, tétrakis,...) sert à dénombrer les groupes complexes : 
bis (diméthylamino) $=$ deux fois le groupe indiqué dans la parenthèse. Ces préfixes sont grecs, sauf le premier (bis et non dis).

c) La série conjonctive, latine (bi, ter, quater....) indique la répétition de systèmes hydrocarbonés cycliques identiques. Par exemple, quaternaphtalène, tercyclopropane.

d) La série distributive, aussi latine (bini, terni, quaterni,...), dénombre les cycles ayant le même nombre de chaînons, mais dans lesquels des chaînons appartiennent à plusieurs cycles. Ainsi, quaternihexalane indique que la molécule comporte quatre cycles de six chaînons, mais un nombre total de chaînons inférieur à 24 .

\section{Numérotage}

Chaque atome de la chaîne carbonée (y compris les hétéro-atomes que renferme cette chaîne) reçoit un indice consécutif, qui servira à indiquer la position des substituants et des doubles et triples liaisons. Les chaînes latérales (chaînes carbonées greffées à la chaîne principale servant à établir le nom fondamental) peuvent être traitées de deux façons. La première façon consiste à les numéroter à part, les substituants s'y rattachant étant alors indiqués au moyen de nombres indexés (primed number).

$$
\begin{aligned}
& \begin{array}{llllllllllll}
1 & 2 & 3 & 4 & 5 & 6 & 7 & 8 & 9 & 10 & 11 & 12
\end{array} \\
& \mathrm{C}_{2} \mathrm{CH}-\mathrm{CH}_{2}-\mathrm{CH}_{2}-\mathrm{CH}-\mathrm{CH}_{2}-\mathrm{CH}_{2}-\mathrm{CH}_{-}-\mathrm{CH}_{2}-\mathrm{CH}_{2}-\mathrm{CH}_{2}-\mathrm{CH}_{2}-\mathrm{CH}_{3} \\
& \mathrm{CHCl}_{2} \mathrm{CH}_{3} \mathrm{CHCl}-\mathrm{CH}_{2}-\mathrm{CH}_{3}
\end{aligned}
$$

1,1-dichloro-1'-chloro-4-ethyl-1"-chloro-7-propyldodecane dichloro-1,1 chloro-l'-éthyl-4 chloro-l"-propyl-7 dodécane

$$
\begin{aligned}
& 3^{\prime} 2^{\prime} 1^{\prime \prime} \mathrm{CH}_{3} \\
& \mathrm{CH}_{3}-\mathrm{CH}_{3}-\mathrm{C}-\mathrm{CH}_{3}
\end{aligned}
$$

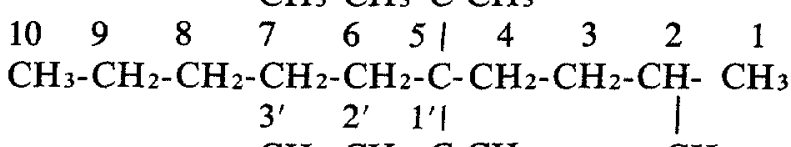

$$
\begin{aligned}
& \mathrm{CH}_{3}-\mathrm{CH}_{2}-\mathrm{C}-\mathrm{CH}_{3} \quad \mathrm{CH}_{3}
\end{aligned}
$$

\section{5,5-bis-1', 1'-dimethylpropyl-2-methyldecane}

bis-diméthyl-I',I'-propyl-5,5 méthyl-2 décane

L'autre méthode consiste à mettre entre parenthèses les chaînes latérales, qui sont alors numérotées de façon indépendante, mais non indexées. Pour les deux exemples donnés ci-dessus, les noms deviennent:

1,1-dichloro-4-(1-chloroethyl)-7-(1-chloropropyl) dodecane dichloro-1,1 (chloro-l éthyl)-4 (chloro-1 propyl)-7 dodécane

et

5,5-bis(1,1-dimethylpropyl)-2-methyldecane bis(diméthyl-1,1 propyl)-5,5 méthyl-2 décane 
Le numérotage de la structure principale est fait de façon que les substituants et les chaines latérales obtiennent des indices de position les plus bas possible. Dans le cas de cycles condensés, le numérotage consécutif ne tient généralement pas compte des chaînons communs à plusieurs cycles. Ces chaînons reçoivent plutôt un indice formé d'un chiffre et d'une lettre. Par ailleurs, les chaînes acycliques greffées à des cycles peuvent être numérotées au moyen de lettres de l'alphabet grec.

Gilles Bélanger

\section{BIBLIOGRAPHIE}

International Union of Pure and Applied Chemistry, Nomenclature of Organic Chemistry, Definitive Rules (1969) Crane, Russak \& Co., New York, 337 p.

LOZAC'H, Noël (1967): la Nomenclature en chimie organique, «Monographies de chimie organique », Paris, Masson, $296 \mathrm{p}$.

Encyclopaedia Universalis, à l'article «chimie».

Dictionnaire encyclopédique Quillet, à l'article «chimie».

Encyclopédie internationale des sciences et des techniques, Larousse, à l'article «chimie».

\section{ERRATUM}

Dans l'article intitulé La nomenclature en chimie minérale (Meta, vol. 27, n 3, p. 295305), une faute d'impression s'est glissée à la page 301, dernier paragraphe. Ce paragraphe devrait commencer ainsi : "Ces préfixes se manient plus facilement en anglais qu'en français. $\mathrm{S}_{2} \mathrm{O}_{7}$, par exemple,... * Par ailleurs stoechiométrique ne prend qu'un seul accent. 